\title{
Analysis of Atrial Fibrillation Laplacian Potential Maps Using Spatial Independent Component Analysis
}

\author{
LY Shyu ${ }^{1}$, YR Lin ${ }^{1}$, SH Jo ${ }^{1}$, CT Tai ${ }^{2}$, WC Hu ${ }^{1}$ \\ ${ }^{1}$ Chung Yuan Christian University, Chung Li, Taiwan \\ ${ }^{2}$ Taipei Veterans General Hospital, Taipei, Taiwan
}

\begin{abstract}
A novel methodology that uses spatial independent component analysis (SICA) to study atria fibrillation (AF) was proposed. Component patterns and their associated time course were extracted from Laplacian potential maps using SICA. Simulation test patterns and potential maps were generated and used to demonstrate the feasibility of the proposed method. It was found that, the time courses that are extracted by SICA could review multiple activations in the simulated potential maps. Additionally, activation duration and number of activations are defined from the extracted time course. Experiment was conducted on eight open chest canines. During sustained AF, the activation durations increased $56.10 \%$ after infusion of Procainamide. On the other hand, the number of activation and the activation block numbers decreased from $2.48 \pm 0.31$ to $1.29 \pm 0.19$ and from $8.96 \pm 0.52$ to $6.41 \pm 1.28$, respectively. The results indicate that when AF occurs, there are multiple wavelets activated spontaneously around the periphery of the atrium. After Procainamide infusion, the numbers of wavelet and the numbers of activation block decreased. These results match the current knowledge of AF and indicate that the proposed technique has significant potential in the analysis of atria fibrillation. Studies will be conducted in the future to further validate its usefulness in clinical diagnosis.
\end{abstract}

\section{Introduction}

Currently, the initiation and sustaining of atria fibrillation (AF) are still under intense investigation. Many studies use high density electrode matrix to acquire electrograms in order to generate potential maps and/or Laplacian potential maps. From these maps the detail spatiotemporal organization in the atria during sustained AF can be studied. However, to review the phenomenon of multiple wavelets that was suggested by Moe, consecutive potential maps are needed and it is very cumbersome to study directly from these maps. Thus, methods were proposed to compress information from multiple potential maps into activation and/or isochronal maps in order to review activity during activation. Due to the nature of the abovementioned methods, related information is accumulated over multiple potential maps into one map. One major problem with these techniques is the lack of information about the time of occurrence of activation. That is, activation and isochronal maps provide detailed spatial information without temporal relationship.

On the other hand, spatial independent component analysis (SICA) has been used in functional magnetic resonance imaging (fMRI) analysis [1-3]. It is found that each independent component pattern that is extracted by the SICA is associated with a time course. And, there is one and only one time course that is closely matched with the time course of experimental tasks. These studies clearly demonstrated the ability of SICA in extracting time course from multiple time-related maps.

It is understood that there are discrepancies between fMRI images and electrogram potential maps. However, they are both related to certain instances of time, for example the onset of task during fMRI study and the initiating of paroxysmal AF in this study. Based on the multiple wavelet theory proposed by Moe, it is hypothesized that, during AF, there are multiple electrical excitations (wavelets) that propagate through their particular conducting pathways. Every pixel on the potential map that is part of this pathway shares similar but delayed activation time course. Thus, the time delay and morphology of time courses is the key in AF analysis. The goal of this study is to investigate and demonstrate the effectiveness of this novel methodology.

\section{Methods}

The ICA method assumes that the relationship, in matrix notation, between observed data $\mathrm{X}$, mixing matrix $\mathrm{M}$ and independent component $\mathrm{C}$ can be expressed as $\mathrm{X}=$ MC. ICA finds the linear transformation $\mathrm{C}=\mathrm{WX}$ to obtain, $c_{i}$, the $i^{\text {th }}$ components in the component matrix, that are as independent as possible in the sense of maximizing some independence measurement functions $F\left(\mathrm{c}_{1}, \ldots, \mathrm{c}_{m}\right)$. When $\mathrm{W}^{-1}=\mathrm{M}$, the observed data can be perfectly reconstructed. 
In the study of McKeown et al [1-3], ICA was used to extract spatially independent patterns from fMRI images. It is found that each component pattern associated with a time course, which is given in the corresponding column of $\mathrm{W}^{-1}$. Additionally, there is one and only one time course that is closely matched with the time course of experimental tasks [1-3].

In our case there is no known time course. However, during sustained AF, each episode has similar electrical excitation propagation pathway that generates a comparable time course. Thus, this study examines the delay and morphology of time course during each activation, instead.

This study uses the JADE algorithm for SICA computation due to the fact that this technique is more effective for data sets with a large number of samples [4]., After successfully separating the time course and component map, z-score was calculated for each component map. Where z-score is defined as.

$$
z_{i}=\frac{x_{i}-\bar{x}}{\sigma}
$$

The z-score information is used to generate blackand-white component maps. For each component map, pixels with z-score less than 2 are reset into white background and pixels with z-score higher than 2 are reset into black. These black-and-white component maps are used in the final analysis in order to reduce the effect of noise.

To demonstrate the ability and effect of spatial ICA in the separation of component maps and time courses, a set of test patterns that consist of three activation patterns, a slow V-shape activation in the middle, a circular activation on the right and a fast circular activation on the lower left are deputed in Figure 1. It is very difficult to identify these patterns from the original maps and component maps directly. On the other hand, the time courses (Figure 1c) confirm the three distinct propagation profiles. Profile one, including time courses 1 to 10 , encloses four activations with different time delay. Profile two, including time courses 11 to 18 , consists of two activations in the same time period. Profile three, including time courses 19 to 39 , have only one activation in the same period. Additionally, when the corresponding component maps of each profile were added together, the activation patterns emerged (Figure 2).

It is known that, during $\mathrm{AF}$, there are multiple activations (reentry) appear simultaneously. The simulation results indicate that the proposed spatial ICA analysis method is feasible in analyzing the propagation of activation in Laplacian potential maps.

Electrograms of eight open chest canines were acquired using a set of 120 unipolar electrodes which were arranged in a 15 by 8 matrix. The electrode matrix was placed on the right atrium epicardium and the upper tip of electrode matrix was placed close to the SVC (superior vena cava). Signals were acquired using Pruka Computerized Cardiac Mapping System (Pruka Engineering, Houston, Texas) with a sampling rate of $1000 \mathrm{~Hz}$. At the beginning of the experiment, electrograms during normal sinus arrhythmia were recorded followed by continuous injection of acetylcholine $(0.5 \mu \mathrm{g} / \mathrm{kg} / \mathrm{min})$. AF was inducted by electrical stimulations. The stimulation interval was $300 \mathrm{~ms}$. When the inducted AF was sustained for more than five minutes, a $28 \mathrm{~s}$ AF electrogram was recorded. Intravenous infusion of Procainamide $(30 \mathrm{mg} / \mathrm{kg})$ started after AF was successfully inducted. The experimental procedure was evaluated and approved by hospital's ethics committee (Taipei Veterans General Hospital, Taipei, Taiwan, ROC).

According to a study by Coronel, the zero crossing of the Laplacian potential map indicates the time of activation. Correspondingly, the zero crossing in the time course indicates the time of activation. The activation duration is defined as the time period between the start of activation and the end of activation. As indicated in Figure 4, the red line indicates the zero crossing in the time course which, in turn, specifies the time of activation. The activation duration is defined as the duration between the two green lines. The two horizontal purple lines indicate the two thresholds for defining the beginning and end of activation duration. The number of activation is defined as the number of zero crossing in the time course.

Self-developed software was implemented using MatLab to calculate the $2^{\text {nd }}$ moment derivative from adjacent electrical activities [5]. The resulting Laplacian potentials were reconstructed into Laplacian potential maps every 2 ms. During AF, a set of 40 Laplacian potential maps were collected for SICA analysis. All the pixel elements in the Laplacian potential map, in each time period, were arranged into one row of the observed data matrix X, as proposed by McKeown et al [1-3]. The SICA computation was done by a MatLab program that was developed based on the JADE code on the website provided by Cardoso [6].

\section{Results}

Figure 3 and 4 illustrate typical Laplacian potential maps, time courses and component maps during NSR and $\mathrm{AF}$, respectively. It can be seen that the time courses during NSR closely resemble each other. Conversely, there is no distinct profile in the time courses during AF. Moreover, the time courses during AF have more number of activations during the same time period. Additionally, the duration of activation is longer during AF.

From results of simulation, the time course of particular component pattern indicates the number of 
activation and time duration when this pattern appears. Thus, the number of activation and activation duration in each time courses were studied and compared before and after the injection of Procainamide during AF.

Table one summarizes results of activation duration comparison. It is found that activation duration after the infusion of Procainamide is significantly longer, increased by $56.1 \%$, than before infusion. The number of activation in the $80 \mathrm{~ms}$ period after the infusion of Procainamide is significantly smaller than those before the infusion, except A17 (Table 2). On average, the number of activation and the activation block numbers decreased from $2.48 \pm 0.31$ to $1.29 \pm 0.19$ and from $8.96 \pm 0.52$ to $6.41 \pm 1.28$, respectively.

Convergence of the ICA analysis for 40 Laplacian potential maps typically took $10 \mathrm{~min}$ on a PC. However, the time it took will not be a significant issue in the near future as long as the method itself is proven to be effective

\section{Discussion and conclusions}

A novel methodology that uses spatial independent component analysis to study atria fibrillation was proposed and evaluated. Component patterns and their associated time course were extracted using SICA based on the JADE algorithm. The results match the current knowledge of Procainamide on AF and indicate that the proposed technique has significant potential in the analysis of atria fibrillation. Studies will be conducted in the future to further validate its usefulness in clinical diagnosis.

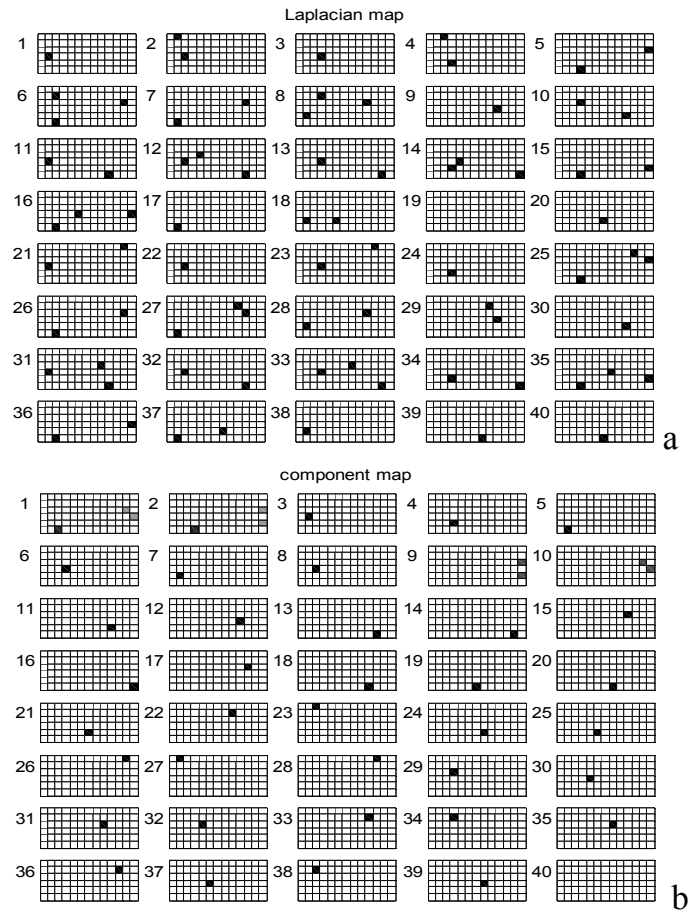

\section{Acknowledgements}

This study was supported by a grant from Nation Science Council, Taiwan, ROC, (NSC-94-2614-E-033026 )

\section{References}

[1] M.J. McKeown, S. Makeig, G.G. Brown, T.P. Jung, S.S. Kindermann, A.J. Bell, T.J. Sejnowski, "Analysis of fMRI Data by Blind Separation Into Independent Spatial Component," Human Brain Mapping, 1998, 6; 160-188.

[2] M.J. McKeown, T.J. Sejnowski, "Independent Component Analysis of fMRI Data: Examining the Assumption," Human Brain Mapping, 1998, 6; 368-372.

[3] M.J. McKeown, "Detection of Consistently Task-Related Activations in fMRI Data with Hybrid Independent Component Analysis," NeuroImage, 2000, 11, 24-35.

[4] J. Cardoso, "High-Order Contrasts for Independent Component Analysis," Neural Comput, 1999, 11, 157-192.

[5] L.Y. Shyu, Y,Y, Hseih, C.T. Tai, T. Kao, W.C. Hu, "Methodology Improvement in Defining Characteristics of Atrial Fibrillation Termination," Computers in Cardiology, 2004, 31, 33- 35.

[6] J. Cardoso, "JADE for real-valued data" Available: $\underline{\text { http://www.tsi.enst.fr/ cardoso/guidesepsou.html }}$

Address for correspondence

Liang-Yu Shyu, Ph.D., Professor

Department of Biomedical Engineering, Chung Yuan University, Chung Li, Taiwan, ROC, 32023

lshyu@be.cycu.edu.tw

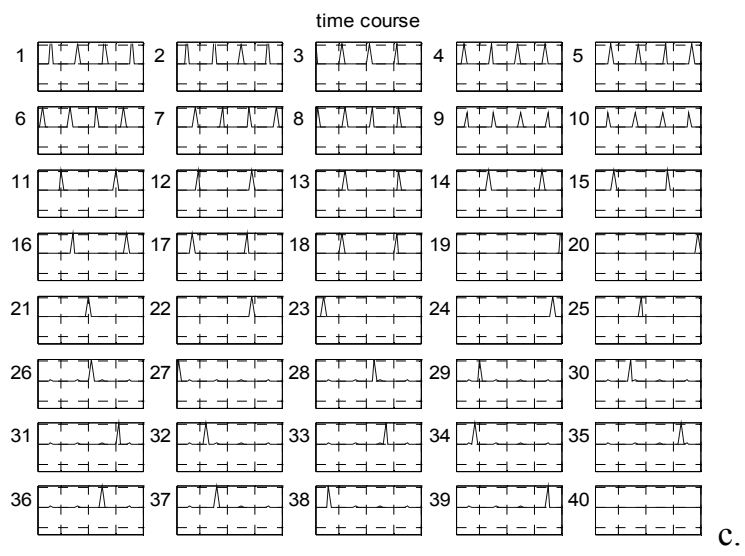

Figure 1. (a) Simulated Laplacian potential maps, (b) component maps and (c) time courses.
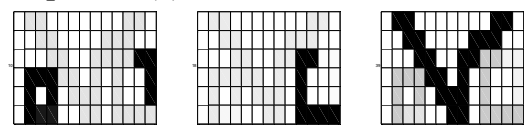

Figure 2. The emerged three activation patterns after adding corresponding component maps of each profile together. 


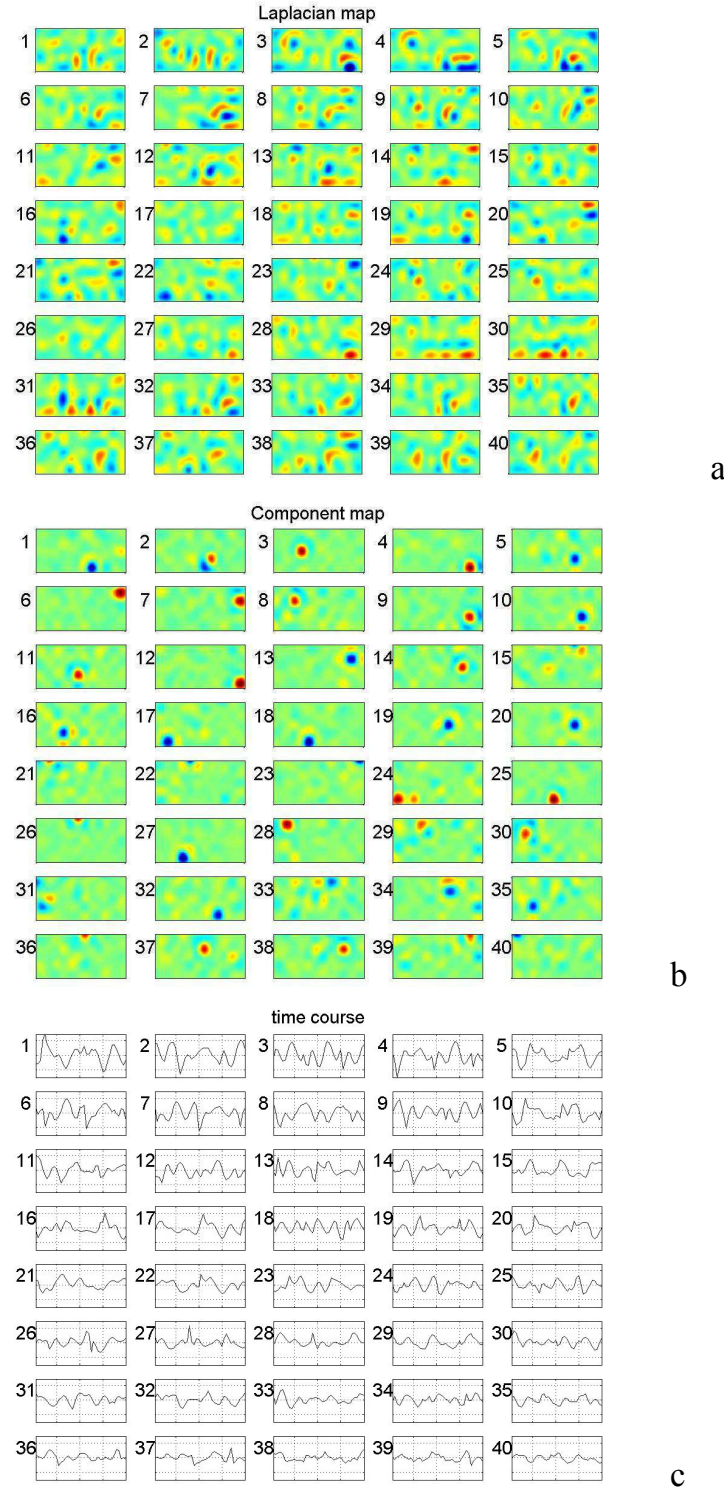

Figure 3. (a) Laplacian potential maps during NSR, (b) component maps and (c) time courses.

Table 1. Activation duration after the infusion of Procainamide is significantly longer than before infusion.

\begin{tabular}{|c|c|c|c|c|}
\hline \multicolumn{5}{|c|}{ Activation duration } \\
\hline & NSR & before & after & P Value \\
\hline A9 & $10.24 \pm 4.37$ & $17.62 \pm 7.93$ & $30.17 \pm 13.53$ & $<0.001$ \\
\hline A13 & $12.00 \pm 8.48$ & $17.63 \pm 7.69$ & $27.04 \pm 12.41$ & $<0.001$ \\
\hline A17 & $12.96 \pm 8.75$ & $21.98 \pm 12.75$ & $32.60 \pm 18.11$ & $<0.001$ \\
\hline A19 & $11.87 \pm 5.09$ & $22.26 \pm 10.74$ & $29.95 \pm 12.71$ & $<0.001$ \\
\hline A21 & $11.62 \pm 4.49$ & $18.94 \pm 8.57$ & $30.27 \pm 16.72$ & $<0.001$ \\
\hline mean & $11.72 \pm 5.95$ & $20.31 \pm 9.26$ & $30.19 \pm 14.74$ & \\
\hline
\end{tabular}

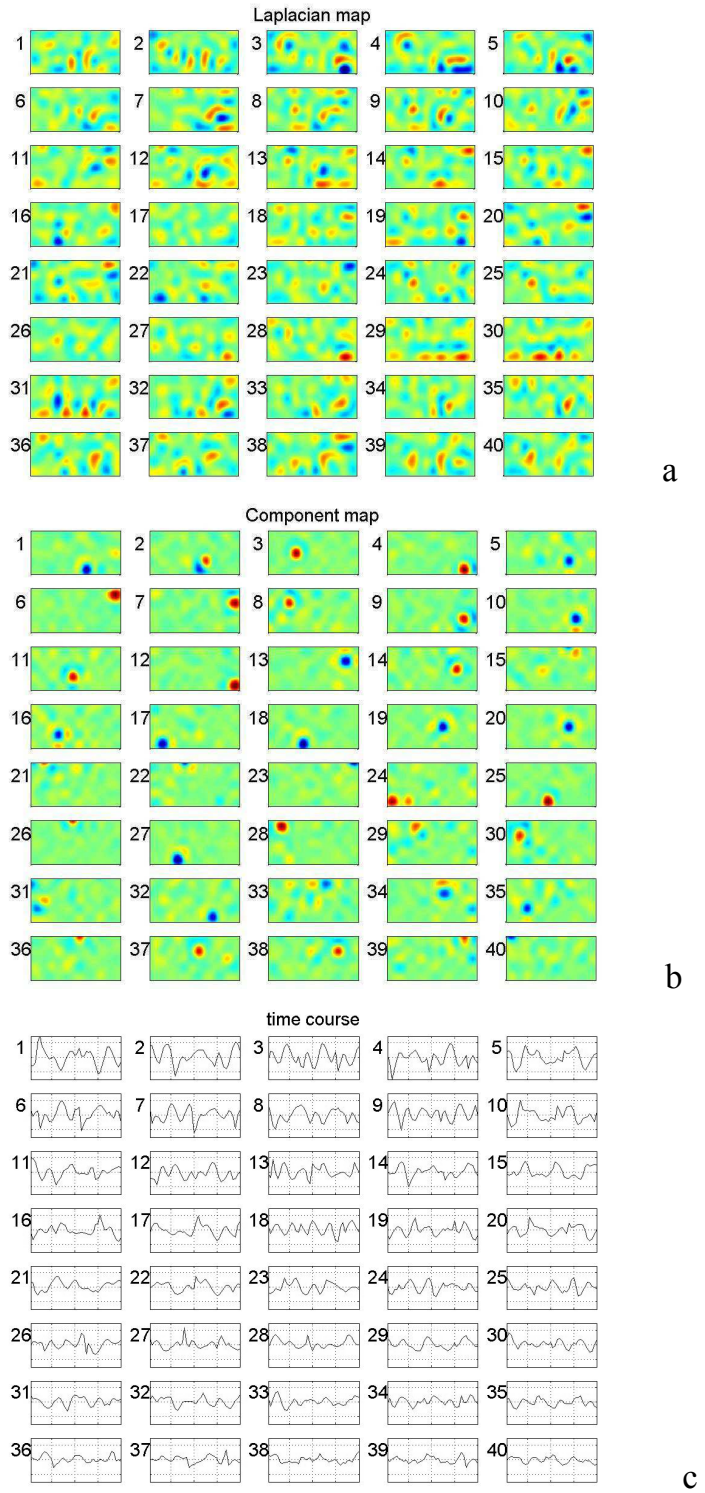

Figure 4. (a) Laplacian potential maps during AF, (b) component maps and (c) time courses.

Table 2. Number of activation in $80 \mathrm{~ms}$ period after the infusion of Procainamide is significantly lower than before infusion, except A17.

\begin{tabular}{|c|c|c|c|c|}
\hline \multicolumn{6}{|c|}{ Number of activation in 80ms } \\
\hline & NSR & before & after & P Value \\
\hline A9 & $1.76 \pm 0.15$ & $2.39 \pm 0.37$ & $1.31 \pm 0.21$ & $<0.001$ \\
\hline A13 & $1.84 \pm 0.17$ & $2.35 \pm 0.24$ & $1.31 \pm 0.21$ & $<0.001$ \\
\hline A17 & $1.86 \pm 0.43$ & $1.22 \pm 0.13$ & $1.34 \pm 0.27$ & 0.2240 \\
\hline A19 & $1.85 \pm 0.19$ & $1.87 \pm 0.16$ & $1.14 \pm 0.08$ & $<0.001$ \\
\hline A21 & $2.08 \pm 0.19$ & $2.82 \pm 0.31$ & $1.36 \pm 0.24$ & $<0.001$ \\
\hline mean & $1.92 \pm 0.18$ & $2.48 \pm 0.31$ & $1.29 \pm 0.19$ & \\
\hline
\end{tabular}

